\title{
Automated Synthesis of Oligosaccharides Provides Access to Chemical Tools and Vaccine Candidates
}

\author{
Lenz Kröck and Peter H. Seeberger
}

\begin{abstract}
Defined, pure oligosaccharides are important tools for biologists to study the structure and function of carbohydrates in biological systems and to create novel diagnostic and therapeutic approaches. Access to reasonable quantities of sufficiently pure oligosaccharides by isolation is difficult and time consuming. Synthetic chemists are therefore called upon to develop straightforward and reliable methods to produce carbohydrates. These molecules are key to the creation of molecular tools and vaccine candidate molecules. A brief overview of the state-of-the-art of automated synthesis is followed by some illustrative examples to highlight applications.
\end{abstract}

Keywords: Automation · Carbohydrate synthesis · Oligosaccharides · Solid-phase

\section{Why Carbohydrate Synthesis?}

The importance of carbohydrates as carriers of information and as key mediators of cellular interactions is now well appreciated.[1] Consequently, interest in studying biological processes that involve different classes of carbohydrates such as glycoproteins, glycolipids, glycosaminoglycans and GPI anchors has drastically increased. Biochemists and biologists interested in specific structural or functional questions often find themselves in a difficult position as the molecules they are trying to study

${ }^{*}$ Correspondence: Prof. Dr. P. H. Seeberger Laboratorium für Organische Chemie der Eidgenössischen Technischen Hochschule (ETH) Zürich

HCl F315

Wolfgang-Pauli-Strasse 10

$\mathrm{CH}-8093$ Zürich

Tel.: + 41446332103

Fax: + 414463311235

E-Mail: seeberger@org.chem.ethz.ch are hard to access. When using isolated carbohydrates, only very small quantities of usually impure molecules are available such that structure-activity relationships are difficult to determine since impurities may be responsible for the effects that are observed. Pure and well-characterized synthetic molecules are of course ideal tools, particularly when incorporated into technology platforms such as carbohydrate microarrays.

\section{What Renders Oligosaccharide Synthesis so Challenging?}

The challenge that lies in the synthesis of oligosaccharides becomes most apparent when comparing sugars to the two other classes of biopolymers that are routinely synthesized by automation. Oligonucleotides and peptides are strictly linear biopolymers while oligosaccharides exhibit highly branched structures. Four nucleic acid and 20 amino acid building blocks are required for synthesis. Mammalian carbohydrates consist of ten monosaccharides. These monosaccharides each can be linked via multiple positions. Furthermore, a new stereogenic center is created in every coupling and results in two possible isomers. Multiple linking positions and stereoisomers result in higher diversity of possible molecular structures as illustrated by the following example:[2,3] The number of theoretically possible structures in case of a hexanucleotide is $4^{6}(=4096)$, for a hexa- peptide $20^{6}$ (= 64 million) and for a hexasaccharide 192 billion.

Theoretically, 224 building blocks would be required to assemble any given mammalian oligosaccharide when considering the ten mammalian monosaccharides and all possible connections and anomers. A recent bioinformatics study revealed that nature only uses a fraction of the possible glycospace: just 36 building blocks suffice to allow for the synthesis of $75 \%$ of mammalian carbohydrate structures. ${ }^{[3]}$

\section{What Challenges Had to $\mathrm{Be}$ Overcome in the Automation of Oligosaccharide Synthesis?}

The automation of oligosaccharide synthesis required us to combine synthetic methodology with a synthesizer robot. Both had to be developed simultaneously and fine tuned to work hand in hand.

\subsection{Synthetic Methodology}

\subsubsection{Synthetic Strategy}

First, a synthetic strategy had to be selected. Automated oligonucleotide and peptide synthesis rely on the solid-phase approach. The main advantage of the solidphase approach lies in the use of excess reagents to drive coupling reactions to completion. Solid-phase peptide and oligonucleotide synthesis is carried out as follows: A growing chain of the biopolymer is synthesized in a strictly linear fashion on a polymeric support. A nucleophile exposed 
on the support is reacted with excess building block (coupling) that carries permanent and temporary protecting groups. Removal of the temporary protecting group (deprotection) reveals a nucleophile that in turn can undergo a coupling step and thereby completes the synthetic cycle. The solid support serves as a handle for washing steps that remove byproducts generated during coupling and deprotection. Upon completion of the synthesis, the material is disconnected from the solid support (cleavage) and purified by HPLC. Permanent protecting groups are generally removed (global deprotection) in the cleavage step. Since the coupling, deprotection and washing steps are repeated, the solid-phase approach lends itself to automation.

Solid-phase oligosaccharide synthesis was first explored in the early 1970s. ${ }^{[4]}$ In solid-supported oligosaccharide synthesis either the sugar carrying the anomeric leaving group (glycosyl donor) or the nucleophile (glycosyl acceptor) can be attached to the solid support (Scheme 1). Both approaches have been explored. ${ }^{[5]}$ The acceptor-bound approach was found to be superior since the glycosyl donor is the more reactive species and is more prone to decomposition. The acceptor-bound approach allows the glycosylating agent to be used in excess. Side products deriving from donor decomposition remain in solution and can be simply washed away. When the donor is bound to the solid phase, decomposition of the limiting reagent would result in an unproductive reaction and diminish the overall yield.

To establish a suitable synthetic methodology based on the acceptor-bound solidphase approach the following issues had to be addressed:

i) development of a linker that is compatible with all synthetic operations (Section 3.1.2) and can be cleaved efficiently (Section 3.1.5)

ii) identification of building blocks required to access the relevant structures (Section 3.1.3) equipped with readily removable protecting groups (Section 3.1.6)

iii) rapid and selective coupling and deprotection conditions (Section 3.1.4)

iv) purification of the final product (Section 3.1.6)

\subsubsection{Solid Support and Linker}

Merrifield's resin ${ }^{[6]}$ was found to be most suitable among the commercially available solid supports. This chloromethylated polystyrene resin also used in peptide synthesis shows the best performance with respect to loading, swelling, solvent and reagent compatibility. The stability towards acids and bases, used in the glycosylation and deprotection steps of the synthetic cycle, is an important advantage.

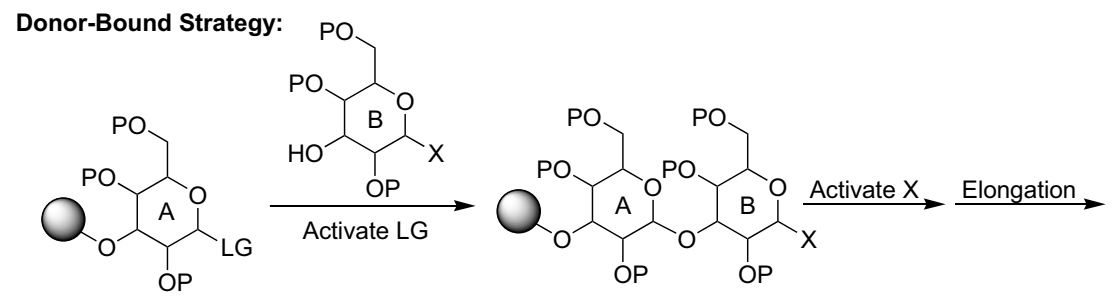

Acceptor-Bound Strategy:

Elongation Deprotection

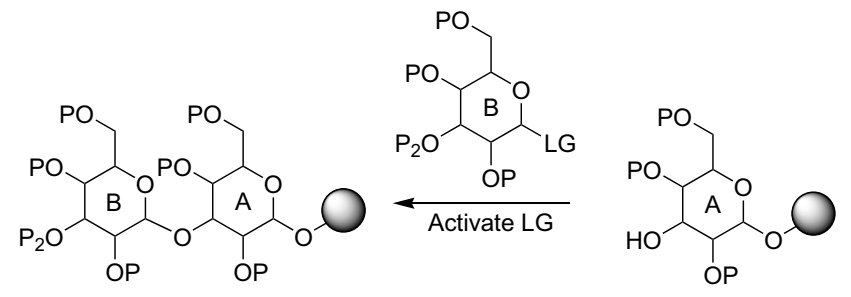

Scheme 1. Donor- and acceptor-bound approach

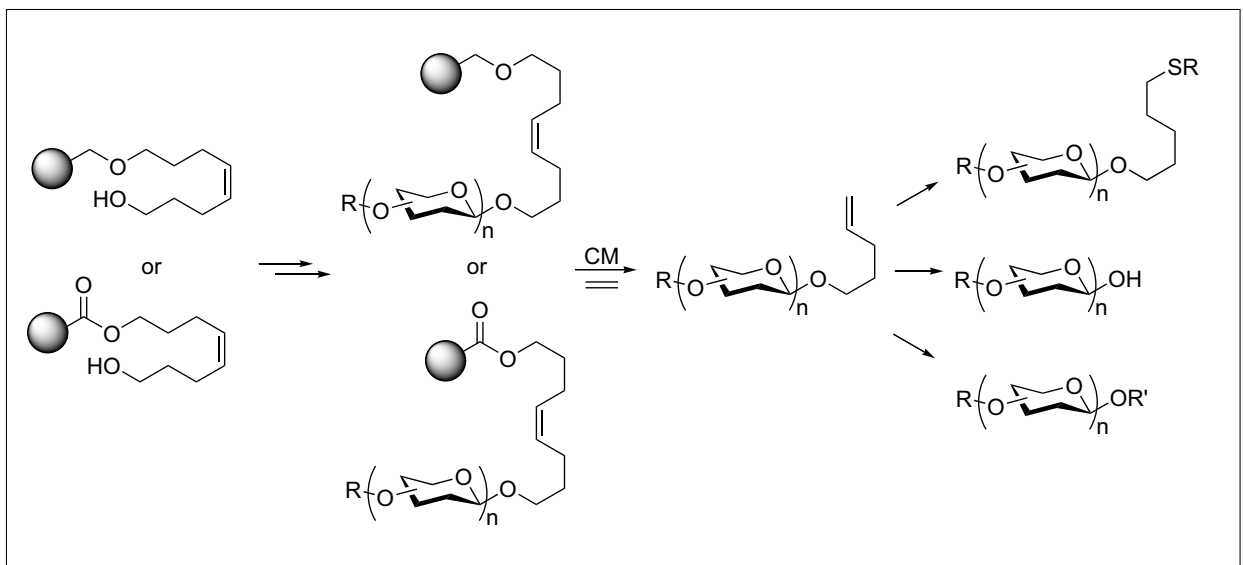

Scheme 2. Octenediol and ester linkers

The linker connects the first unit of the growing oligosaccharide chain to the solid support. It has to remain intact during the synthesis but has to be cleaved efficiently, selectively and under relatively mild conditions at the end of the synthesis. Furthermore, the cleaved linker should allow attachment to a protein carrier or a chip surface and ideally enable release of a free reducing end (Scheme 2).

A linker containing a double bond fulfills these prerequisites. The double bond was incorporated in form of an octenediol linker that is connected to the support either by an ether ${ }^{[7]}$ or an ester ${ }^{[8]}$ linkage. The product is released by cross metathesis using Grubbs catalyst in presence of ethylene without affecting the protecting groups. Cleavage results in the oligosaccharide release in form of a $n$-pentenyl glycoside. The terminal olefin can be chemically modified to connect the sugar to a protein carrier or chip surface. ${ }^{[9]}$ Removal of the pentenyl glycoside furnishes the free reducing sugar. The octenediol linker works well although it precludes the use of electrophiles and therefore limits the glycosylation methods that can be used.

\subsubsection{Building Blocks}

The identification and synthesis of the monosaccharide building blocks required for the automated synthesis is currently the most laborious and time consuming task. Nevertheless it is a very important aspect in the development of a commercial synthesizer. Once the most suitable building blocks have been identified and tested, their large scale synthesis will be commercialized as is the case for peptide and oligonucleotide building blocks.

A building block for oligosaccharide synthesis generally carries permanent and temporary protecting groups. The anomeric position is equipped with a leaving group. In our approach benzyl ethers (Bn), pivaloyl esters (Piv), azides and N-trichloroacetyl (TCA) groups serve as permanent protecting groups -i.e. those groups will only be removed during global deprotection at the end of the synthesis (Scheme 3). 9-Fluorenylcarbyloxymethyl (Fmoc), levulinoyl 
(Lev), acetyl (Ac) and silyl-protecting groups were chosen for temporary protection. These groups are cleaved upon coupling to allow for elongation of the growing biopolymer.

The development of anomeric leaving groups has been the focus of carbohydrate chemistry for decades. ${ }^{[10]}$ For the automated approach we found glycosyl trichloroacetimidates $^{[11]}$ and phosphates ${ }^{[12]}$ the most suitable choice. Both classes of compounds are reasonably stable - they can be stored longterm in the freezer without decomposition and both compounds can be activated by TMSOTf addition - a caustic, but nontoxic reagent. Most importantly, both glycosylating agents generally result in high yielding and selective glycosylations.

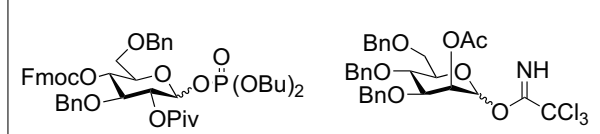

Scheme 3. Representative examples of building blocks

Alternative protecting and leaving groups can be envisioned. The examples described above can be seen as a starting point and certainly will be further developed in the future.

\subsubsection{Synthetic Cycle}

The assembly of oligosaccharides involves a two-step synthetic cycle consisting of glycosylation and deprotection. Between those two steps the resin is washed thoroughly to remove any byproducts. In the glycosylation step a solid support bound nucleophile is reacted with the anomeric position of the incoming building block to furnish a glycosidic linkage. In the initial glycosylation, the incoming nucleophile is the hydroxyl of the linker to anchor the growing biopolymer to the solid support. Selective removal of a temporary protecting group furnishes a hydroxyl group as nucleophile for the next coupling (Scheme 4).

In the glycosylation step the building blocks are commonly used twice in fivefold excess with respect to the solid phase bound nucleophile. These so-called 'double couplings' have been shown to result in coupling yields that exceed $98 \%$. In case of glycosyl phosphates a five-fold excess of TMSOTf as activating reagent is used while glycosyl trichloroacetimidates only require 0.75 equiv. of this activating agent. The temperature for coupling reactions varies from $-15{ }^{\circ} \mathrm{C}$ to room temperature.

Fmoc is currently used as the standard temporary protecting group. ${ }^{[8]}$ This carbamate is cleaved with piperidine $(20 \%$

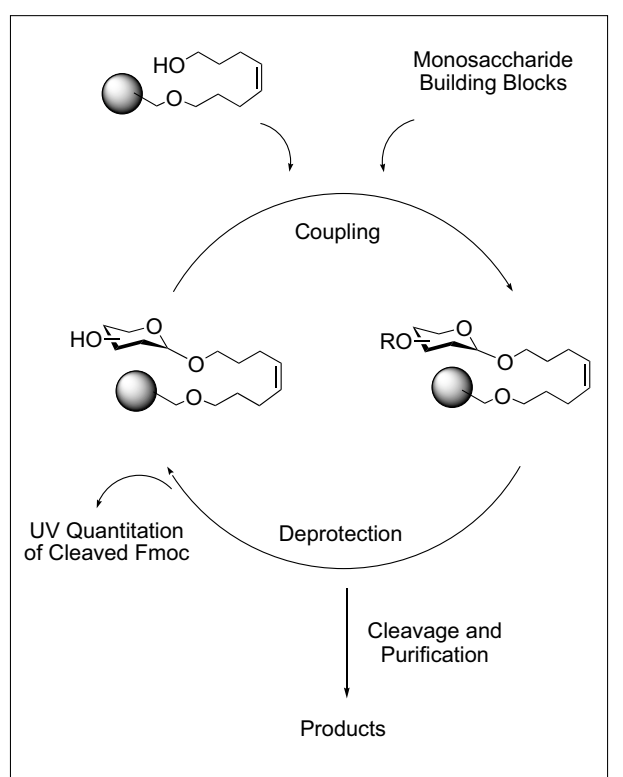

Scheme 4. Coupling cycle of automated oligosaccharide synthesis

in DMF). The main advantage of Fmoc in comparison to acetate or levanulinyl esters lies in the fast and mild deprotection conditions and in the UV-activity of the dibenzofulvene byproduct generated during deprotection. Coupling efficiency can be assessed by the UV absorbance of the deprotection solution.

\subsubsection{Cleavage from the Resin}

After the coupling cycles have been completed the protected oligosaccharide has to be removed from the solid support. Currently this task is achieved by olefin cross metathesis. The resin is transferred to a round-bottom flask and is treated overnight with Grubbs catalyst under an atmosphere of ethylene. The liberated products are obtained in the form of $n$-pentenyl glycosides that can be separated from the catalyst by filtration. At this point the result of the synthesis can be assessed by HPLC-mass spectrometry (LC-MS) using a small aliquot of the product. Unwanted side-products are removed from the fully protected oligosaccharide by HPLC, since separation is significantly easier than at the fully deprotected stage. Nevertheless, about $40-50 \%$ of the desired product is lost during the HPLC purification. Although recovery rates are similar to those for peptides purification further improvements of the purification protocols have to be achieved.

\subsubsection{Global Deprotection and \\ Purification of the Final Product}

All protecting groups have to be removed from the oligosaccharide to afford the final product. Depending on the nature of the protecting groups one or more transformations are required. When N-trichloroacetyl groups were used as permanent amine protecting groups the corresponding naturally occurring $\mathrm{N}$-acetyl moieties are procured by reduction with zinc in the presence of acetic acid. The commonly used benzyl ethers and any esters are removed by Birch reduction using sodium in liquid ammonia. Alternatively, base-assisted ester cleavage followed by palladium-catalyzed hydrogenolysis has been applied. This protocol results in reduction of the $n$-pentenyl residue that has to be modified prior to hydrogenolysis in order to allow attachment of the oligosaccharide to surfaces. ${ }^{[9]}$

The unprotected sugar finally has to be prepared for use in biological studies. It is passed through size exclusion columns and dialyzed to remove any salts. Characterization relies on full analysis by ${ }^{1} \mathrm{H}$-, ${ }^{13} \mathrm{C}-\mathrm{NMR}$ and mass spectrometry. Once the synthesis process has been streamlined and has proven to be reliable a less thorough analysis e.g. by mass spectrometry only may become sufficient.

\subsection{Synthesizer}

The synthesizer has to execute the synthetic cycle including coupling, deprotection and washing steps. Initially, a modified ABI peptide synthesizer was used (Fig.). ${ }^{[13]}$ The modification enabled temperature control of the reaction vessel as the coupling steps generally take place below $0{ }^{\circ} \mathrm{C}$. The machine uses argon pressure to drive reagent solutions and solvents through Teflon lines and solenoid valves that are opened and closed to control the amount of reagent dispensed. The building blocks are stored in plastic vessels. Delivery of the building block solutions takes place via a needle that punches a hole into the container. The solution then is delivered by argon pressure to the reaction vessel. This vessel is a doublejacketed glass tube with a frit in the bot-

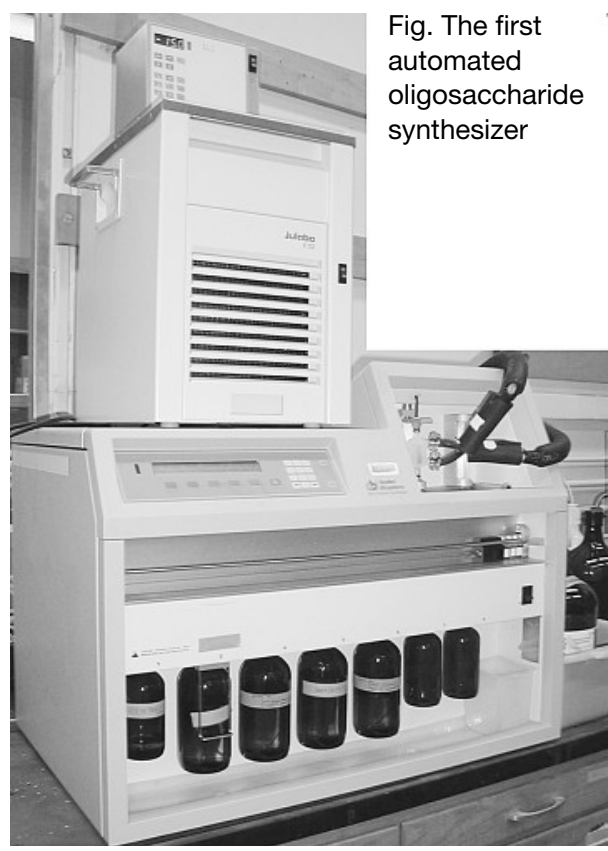


tom. The double jacket is used to circulate a cryogenic fluid that allows for cooling of the reaction vessel. The frit in the vessel retains the solid phase in the vessel while washing solutions can be filtered away. The first prototype required manual adjustment of the temperature as the peptide synthesizer did not communicate with the cryostat. A syringe pump-driven oligosaccharide synthesizer has been developed to overcome this limitation and to have more freedom with regards to the chemistry that can be used.

\section{Automated Oligosaccharide Synthesis: Beyond the Proof of Principle?!}

Three examples of automated syntheses are discussed in order to illustrate the current state of the art of our system.

\subsection{The First Automated Synthesis}

The first automated oligosaccharide synthesis was reported by our group in $2001^{[13]}$ using a phytoalexin elicitor $\beta$-glucan dodecasaccharide as example. This elicitor glycan triggers soybean plants to release antibiotic phytoalexins as a defense mechanism against fungi which exhibit the $\beta$-glucan.

The molecule had been previously prepared in solution ${ }^{[14]}$ and on solid support ${ }^{[15]}$ and served as a benchmark for our early automation endeavors. The synthesis was accomplished using two glycosyl-phosphate building blocks: a monosaccharide and a disaccharide that were alternately used for coupling. The levulinoyl ester served as temporary protecting group. Therefore, real-time monitoring of the coupling yields was impossible. This synthesis was an important milestone. Nevertheless, the synthetic challenge imposed by the branching of the structure was circumvented using disaccharide building blocks, exclusively trans-glycosidic linkages were established and the final compound was not deprotected (Scheme 5).

\subsection{Synthesis of Blood Group Determinants and Tumor-Associat- ed Antigens}

Lewis ${ }^{\mathrm{y}}$ hexasaccharide and $\mathrm{Le}^{\mathrm{y}}-\mathrm{Le}^{\mathrm{x}}$ nonasaccharide are tumor markers that are currently under evaluation as anti-cancer vaccines. ${ }^{[16]}$ Their branched structures imposed a serious challenge to our approach and gave us the opportunity to improve the overall protocol.[7] The resin was modified to include an ester moiety between the linker and the resin. The modified linker enabled faster and more reliable cleavage, but at the same time precluded the use of temporary acetyl protecting groups. As the structures contain branching points a

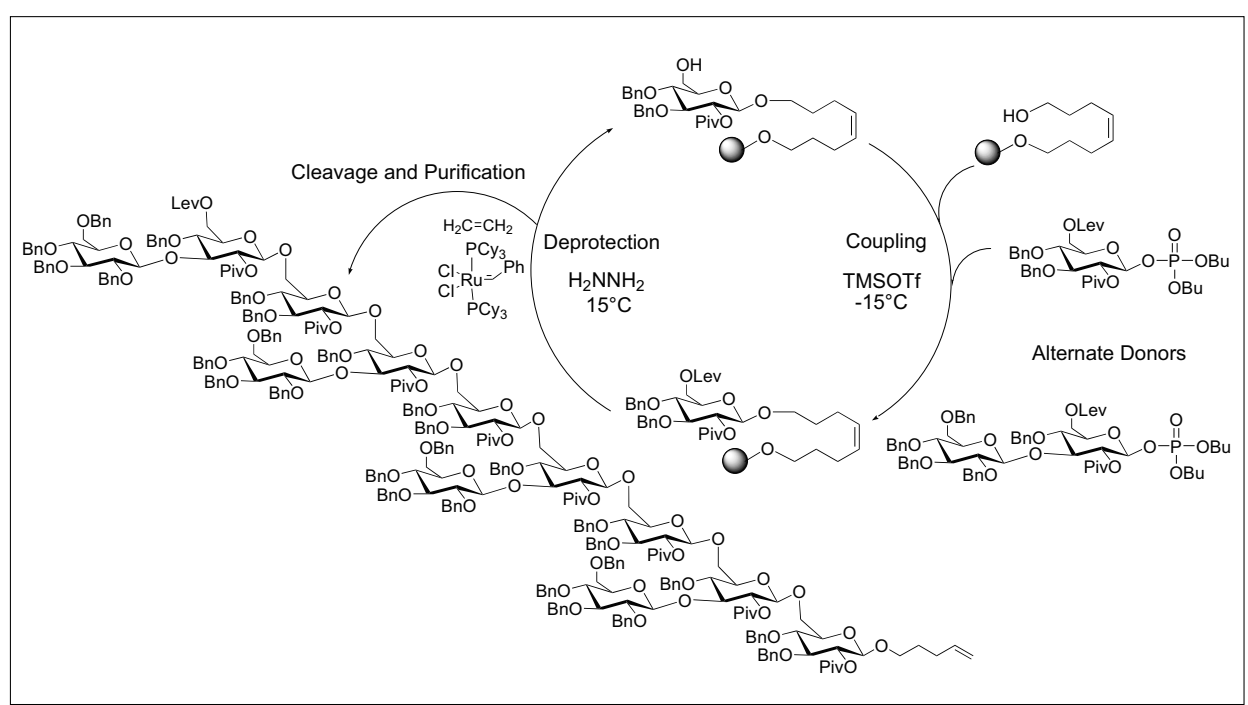

Scheme 5 . Automated synthesis of the $\beta$-glucan dodecamer

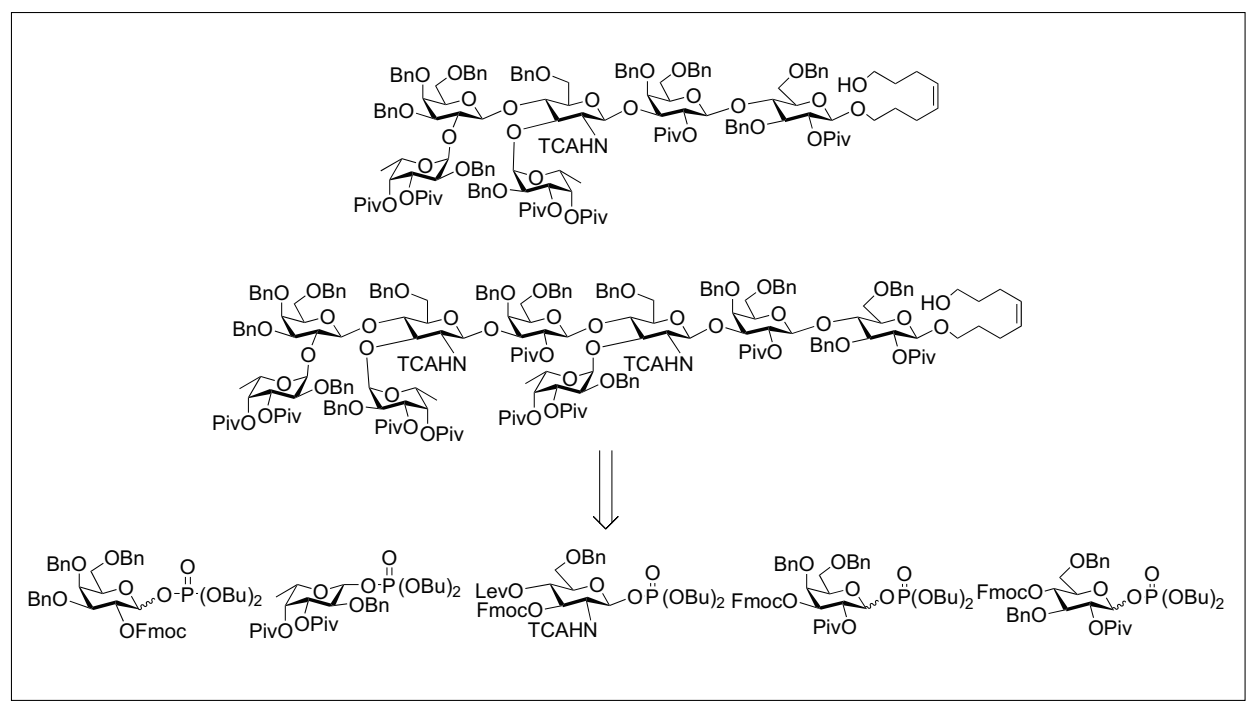

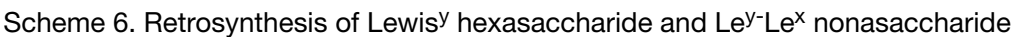

second protecting group orthogonal to the levulinoyl group used in the previous syntheses was required. Fmoc was identified as the optimal choice since it can be cleaved in presence of levalinoyl protection. Fmoc cleavage can be used to monitor the coupling yields, a feature that was of particular importance at the development stage of our synthetic system.

The Lewis ${ }^{\mathrm{y}}$ hexasaccharide was assembled from five different building blocks in an isolated overall yield of $10 \%$. $\mathrm{Le}^{\mathrm{y}}-\mathrm{Le}^{\mathrm{x}}$ nonasaccharide was assembled from the same five building blocks. The synthesis required nine glycosylations, eight deprotections and one cleavage and purification step. The isolated overall yield of the synthesis was $6.5 \%$ (Scheme 6). Given the size and complexity of the molecule, the result underscores the power of our methodology.

Though fully protected, the target oligosaccharides were obtained in overall yields comparable or better than previous solution-phase syntheses, but in a fraction of the time previously required.

\subsection{A First Application of Auto- mated Oligosaccharide Synthesis - An Anti-Toxin Malaria Vaccine Candidate}

Automated oligosaccharide synthesis holds great potential to accelerate the assembly of oligosaccharides to be used in conjugated vaccines. An illustrative first example from our laboratories is the use of automated oligosaccharide synthesis in the construction of a molecule currently in preclinical evaluation as an anti-toxin malaria vaccine candidate. ${ }^{[17]}$

Malaria infects currently $5 \%$ of the world's population, resulting in 100 million clinical cases and 3 million deaths annually. We demonstrated that vaccination with anti-toxin malaria vaccine candidate 
can protect mice from death caused by malaria parasites. ${ }^{[18]}$

The oligosaccharide part of the vaccine candidate was synthesized in a semi-automated fashion as the $\alpha$-linkage between inositol and glucosamine was deemed too challenging for automation. A tetra-mannosyl fragment was synthesized by automation and joined to a disaccharide that was obtained by solution phase synthesis. The oligosaccharide was conjugated to a carrier protein to obtain the vaccine candidate (Scheme 7).

In this case solution-phase synthesis can provide larger amounts of the oligosaccharide, but automated synthesis allows for fast access. This feature will be instrumental in future studies where fast access to structurally diverse oligosaccharide libraries is required.

\section{Conclusion and Outlook}

Automated oligosaccharide synthesis to date has provided access to a broad range of biologically relevant oligosaccharides - three examples have been highlighted in this review.

We were able to address the key challenges towards a general approach of automated oligosaccharide synthesis. Still, there is ample room for improvement. In particular, the steps following the assembly of the structure like cleavage, purification and deprotection have to be streamlined. Further challenges like difficult linkages and complex sequences remain to be tackled. Overall we are confident that our approach will become a powerful tool for glycomics research.

\section{Acknowledgements}

We gratefully acknowledge the ETH Zürich and the Swiss National Science Foundation (SNF, grant 200020 - 109555) for financial support. We thank all of the coworkers that have made the automated carbohydrate synthesizer a reality during the past nine years. Their names are listed in the references. We thank the Roche Research Foundation (Doctoral stipend for L. K.).

Received: October 24, 2007

[1] A. Varki, R. Cummins, J. Esko, H. Freeze, G. Hart, J. Marth, 'Essentials of Glycobiology', Cold Spring Harbor Laboratory Press, New York, 1999.

[2] R. A. Laine, Glycobiology 1994, 4, 759.

[3] D. B. Werz, R. Ranzinger, S. Herget, A. Adibekian, C.-W. von der Lieth, P. H. Seeberger, ACS Chem. Biol. 2007, 2, 685.

[4] J. M. J. Frechet, C. Schuerch, J. Am Chem. Soc. 1971, 93, 492.

[5] P. H. Seeberger, W. C. Haase, Chem. Rev. 2000, 100, 4349.

[6] R. B. Merrifield, Angew. Chem. Int. Ed. Engl. 1985, 24, 799.

[7] R. B. Andrade, O. J. Plante, L. G. Melean, P. H. Seeberger, Org. Lett. 1999, 1, 1811.

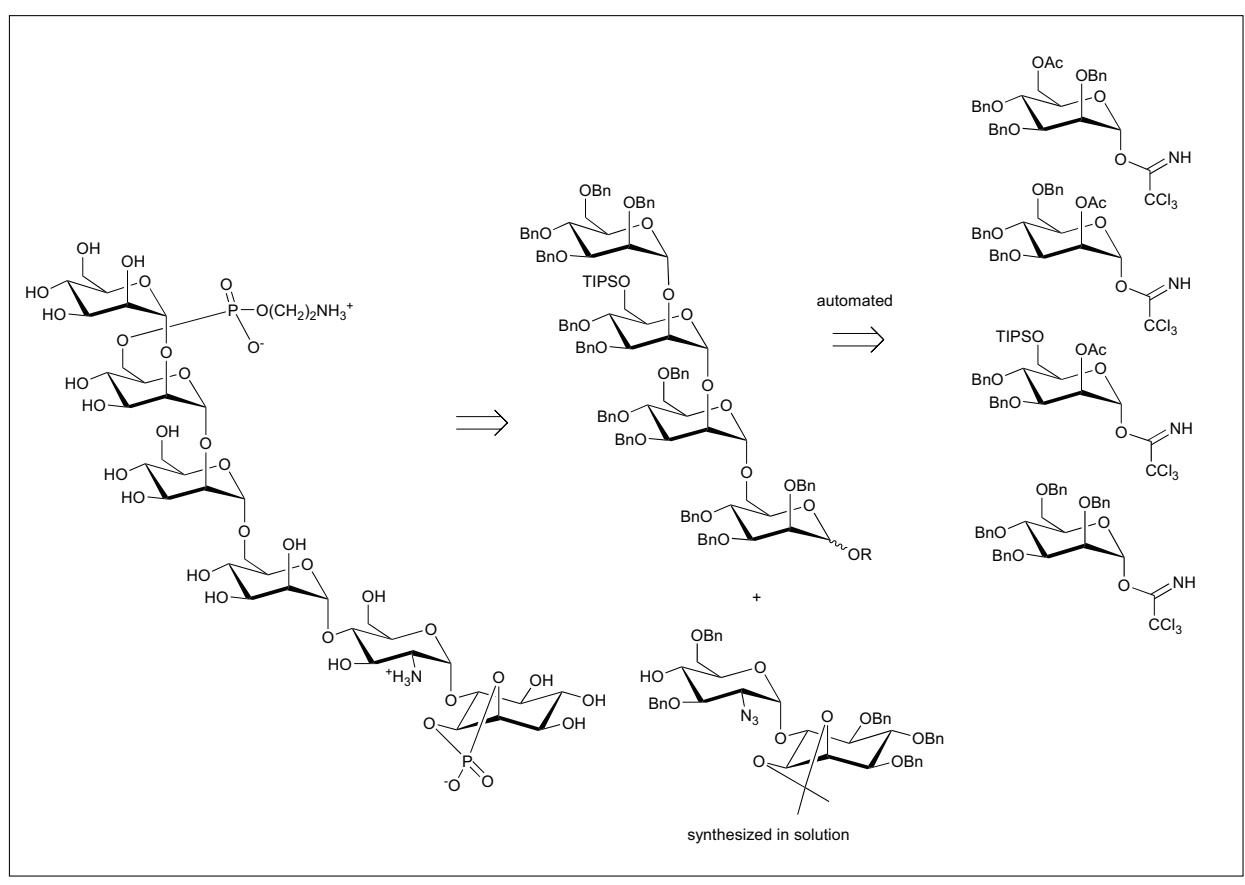

Scheme 7. Retrosynthesis of the malaria vaccine candidate. The disaccharide is synthesized in solution, the tetrasaccharide by automation from four building blocks.

[8] K. R. Love, P. H. Seeberger, Angew. Chem. Int. Ed. 2004, 43, 602.

[9] T. Buskas, E. Soederberg, P. Konradson, B. Fraser-Reid, J. Org. Chem. 2000, 65, 958.

[10] K. Toshima, K. Tatsua, Chem. Rev. 1993, 93, 1503.

[11] R. R. Schmidt, W. Kinzy, Adv. Carbohydr. Chem. Biochem. 1994, 50, 21.

[12] O. J. Plante, E. R. Palmacci, R. B. Andrade, P. H. Seeberger, J. Am. Chem. Soc. 2001 , 123, 9545.

[13] O. J. Plante, E. R. Palmacci, P. H Seeberger, Science 2001, 291, 1523.

[14] P. Fugedi, W. Birberg, P. J. Garegg, A Pilotti, Carbohydr. Res. 1987, 164, 297.
[15] K. C. Nicolaou, N. Winssinger, J. Pastor, F. DeRoose, J. Am. Chem. Soc. 1997, 119 , 449.

[16] G. Ragupathi, P. P. Deshpande, D. M. Coltart, H. M. Kim, L. J. Williams S. J. Danishefsky, P. O. Livingston, Int. J. Cancer 2002, 99, 207.

[17] M. C. Hewitt, D. A. Snyder, P. H Seeberger, J. Am. Chem. Soc. 2002, 124 13434.

[18] L. Schofield, M. C. Hewitt, K. Evans, M. A. Siomos, P. H. Seeberger, Nature 2002, 418,785 . 